Corrigendum

\title{
Corrigendum to "Development and Application of an UHPLC-MS/MS Method for Comparative Pharmacokinetic Study of Eight Major Bioactive Components from Yin Chen Hao Tang in Normal and Acute Liver Injured Rats"
}

\author{
Yun Wang, ${ }_{1}$ Xinrui Xing, ${ }^{2}$ Yan Cao, ${ }^{2}$ Liang Zhao, ${ }^{3}$ Sen Sun, ${ }^{3}$ Yang Chen, ${ }^{2}$ Yifeng Chai $\mathbb{D}^{2}$ \\ Si Chen ${ }^{(D)}{ }^{4}$ and Zhenyu Zhu ${ }^{2}{ }^{2}$ \\ ${ }^{1}$ Hebei Institute for Drug Control, No. 16 Fuqiang Street, Shijiazhuang 050011, China \\ ${ }^{2}$ School of Pharmacy, Second Military Medical University, No. 325 Guohe Road, Shanghai 200433, China \\ ${ }^{3}$ Department of Pharmacy, Eastern Hepatobiliary Surgery Hospital, Shanghai 200438, China \\ ${ }^{4}$ Postdoctoral Research Workstation, 210th Hospital of the Chinese People's Liberation Army, Dalian 116021, China
}

Correspondence should be addressed to Si Chen; caroline-sisi-chen@hotmail.com and Zhenyu Zhu; zzyzyfzhu@163.com

Received 29 November 2018; Accepted 2 December 2018; Published 16 December 2018

Copyright (C) 2018 Yun Wang et al. This is an open access article distributed under the Creative Commons Attribution License, which permits unrestricted use, distribution, and reproduction in any medium, provided the original work is properly cited.

In the article titled "Development and Application of an UHPLC-MS/MS Method for Comparative Pharmacokinetic Study of Eight Major Bioactive Components from Yin Chen Hao Tang in Normal and Acute Liver Injured Rats" [1], the Authors' Contributions section was missing and should be added as follows:

\section{Authors' Contributions}

Yun Wang and Xinrui Xing contributed equally to this work.

\section{References}

[1] Y. Wang, X. Xing, Y. Cao et al., "Development and application of an UHPLC-MS/MS method for comparative pharmacokinetic study of eight major bioactive components from yin chen hao tang in normal and acute liver injured rats," Evidence-Based Complementary and Alternative Medicine, vol. 2018, Article ID 3239785, 12 pages, 2018. 


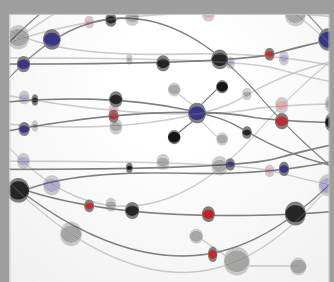

The Scientific World Journal
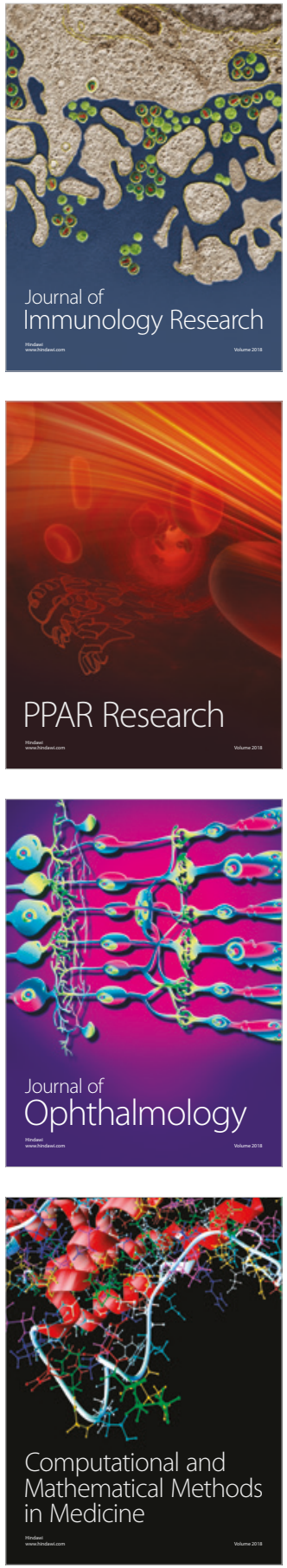

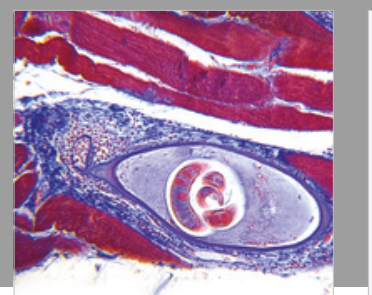

Gastroenterology Research and Practice

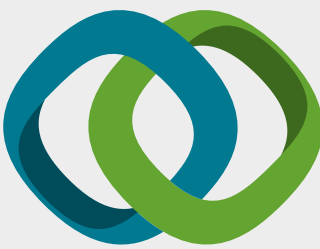

\section{Hindawi}

Submit your manuscripts at

www.hindawi.com
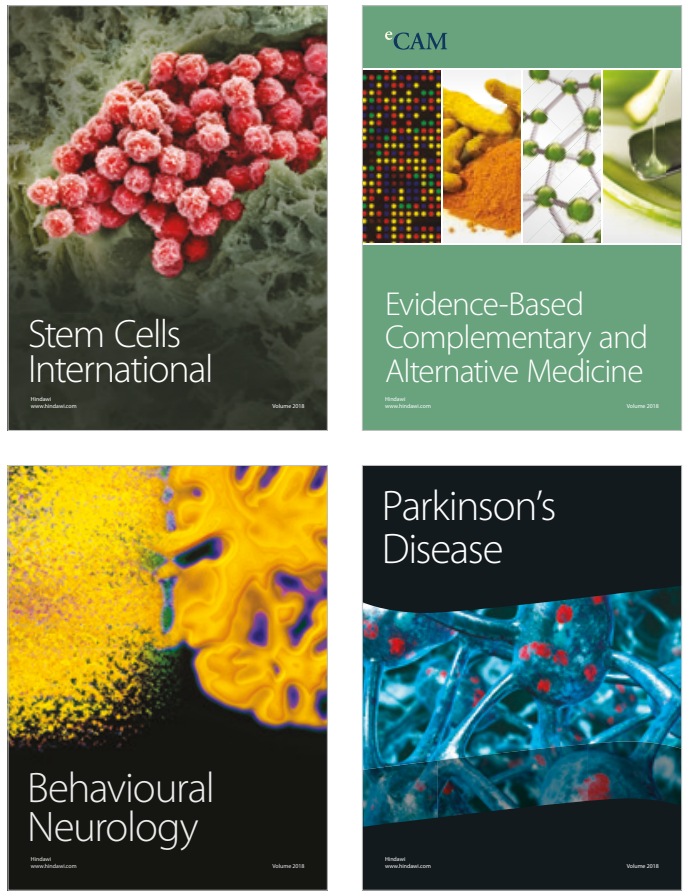

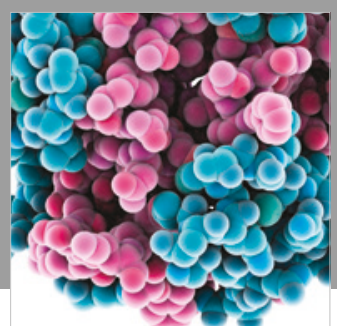

ournal of

Diabetes Research

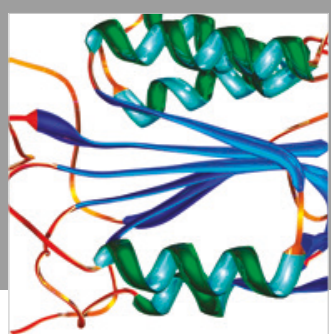

Disease Markers
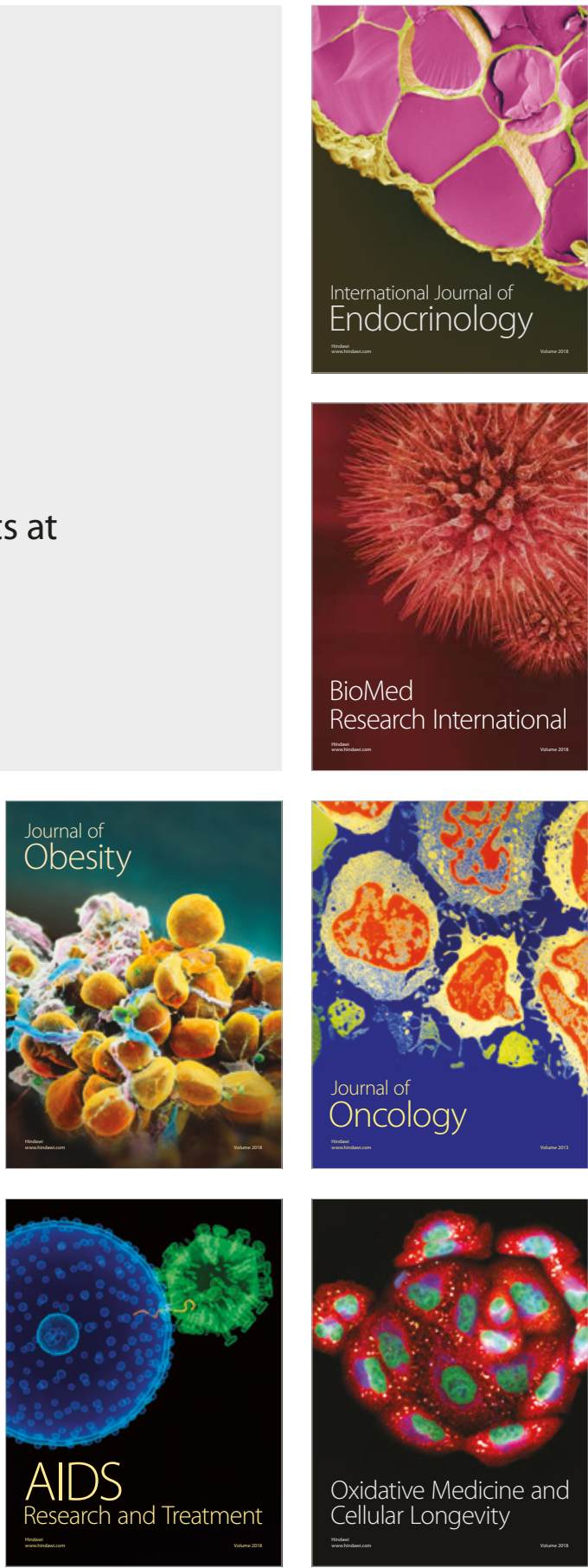